\title{
Environmental attitudes and preference for wetland conservation in Malaysia
}

\begin{abstract}
The incorporation of latent psychological factors in wetland valuation studies may improve our understanding of why some people value ecosystem services while others do not. This article focuses on public preferences for enhanced protection of the Setiu Wetland in Malaysia and explores the influence of environmental attitude on preference and the willingness to pay (WTP) for wetland conservation. The study reported here employs a discrete choice experiment to investigate household's WTP for a set of wetland attributes. A scale-adjusted latent class (SALC) model is applied to identify a latent preference structure combining choice attributes with attitude measures derived from the New Ecological Paradigm (NEP). We identified four NEP components in the respondent population to integrate with SALC model, and this revealed four latent classes and two scale classes which varied in their preferences. Class 1 was largely against wetland protection, although it showed a flooding preference, and was more likely to be 'Anthropocentric' but less likely to be 'Biocentric'. Class 2 had a positive preference for all attributes, and was more likely to be 'Biocentric'. Class 3 did not refer to any of the NEP components. A handful of responses in Class 4 were respondents more likely to be in the 'Risk of overuse' group and less likely to be 'Anthropocentric'. The result suggests that natural resource managers need to evaluate people's concerns over environmental protection to understand potentially conflicting views across populations.
\end{abstract}

Keyword: Wetland conservation; Environmental attitudes; Economic valuation; Scaleadjusted latent class model 